\title{
UM ESTUDO ECOLÓGICO DE TUBERCULOSE NA POPULAÇÃO PRIVADA DE LIBERDADE DA REDE REGIONAL DE ATENÇÃO À SAÚDE 11
}

Sarah da Silva Sá, Gabriel Carlos Leite, Thalita Rolleigh da Silva, Fernando de Moura Moreira, Daniela Tereza Ascencio Russi.

Universidade do Oeste Paulista - UNOESTE, Faculdade de Medicina, Presidente Prudente, SP. E-mail: sarahssa12@gmail.com.

\section{RESUMO}

A População Privada de Liberdade (PPL) é uma população de alto risco para a Tuberculose, sendo que, no Brasil, a incidência da doença no sistema penitenciário é 28 vezes maior que na população em geral. $O$ objetivo deste estudo foi verificar, dentre os casos notificados na Rede Regional de Atenção à Saúde (RRAS) 11 (DRS Presidente Prudente), a ocorrência de Tuberculose na PPL em comparação ao restante da população. Trata-se de um estudo ecológico, quantitativo, utilizando registros do Sistema de Informação de Agravos de Notificação (SINAN) dos casos confirmados de Tuberculose, na Macrorregião de Saúde de Notificação RRAS 11, no período entre 2012 a 2016. Do total de casos notificados, 59,66\% corresponderam a PPL, sendo esta predominante masculina com faixa etária entre 25 a 34 anos de idade. Conclui-se que a tuberculose continua sendo um desafio a ser superado na RRAS 11, principalmente em meio à população prisional.

Palavras-chave: Tuberculose, pessoas privadas de liberdade, prisões, saúde pública, incidência.

\section{AN ECOLOGICAL STUDY OF TUBERCULOSIS IN THE PRISON POPULATION OF REGIONAL HEALTH CARE NETWORK 11}

\begin{abstract}
The prison population (PPL) is a high-risk population for Tuberculosis, and in Brazil the incidence of the disease in the penitentiary system is 28 times higher than in the general population. The objective of this study was to verify the occurrence of Tuberculosis in prison population in relation to the rest of the population, among the cases reported in the Regional Health Care Network (RRAS) 11 (DRS Presidente Prudente). This is an ecological, quantitative study using records from the Notification Disease Information System (SINAN) of confirmed cases of Tuberculosis in the RRAS 11, in the period between 2012 and 2016. Among all of the reported cases, 59.66\% corresponded to the PPL, being predominantly male with an age range between 25 and 34 years of age. It is concluded that tuberculosis remains a challenge to be overcome in the RRAS 11, especially among the prison population.
\end{abstract}

Keywords: Tuberculosis, persons deprived of liberty, prisons, public health, incidence. 


\section{INTRODUÇÃO}

A tuberculose é considerada uma das mais antigas doenças infecciosas da humanidade e, embora passível de um efetivo tratamento, permanece na atualidade como um importante problema de saúde pública mundial, em virtude da ampla dispersão geográfica, emergência de casos multirresistentes e coinfecção com HIV (Lonnroth, 2010). Estima-se que, no ano de 2010, ocorreram no mundo aproximadamente 8,8 milhões de casos novos, 1,1 milhão de óbitos entre indivíduos não portadores de HIV e 400000 óbitos entre pessoas soropositivas para HIV (WHO, 2011).

Sabe-se que cerca de $95 \%$ dos casos de Tb ocorrem no terceiro mundo, sendo que aí ocorrem 98\% dos óbitos (WHO, 1998).

Em 1993, a Organização Mundial da Saúde (OMS) declarou a situação da tuberculose como estado de urgência. Criou-se, então, o programa "STOP TB" que reúne instituições de alto nível científico e/ou poder econômico, tais como: a Organização Mundial da Saúde, o Banco Mundial, o Centers for Disease Control (CDC)- Atlanta, International Union Against Tuberculosis and Lung Disease (IUATLD), Royal Netherlands Tuberculosis Association (RNTA) e American Thoracic Association (ATA) (WHO, 1998).

Em pessoas privadas de liberdade, a Tuberculose constitui um importante problema de saúde pública. No Brasil, a incidência da doença no sistema penitenciário é 28 vezes maior que na população em geral (Navarro, PD, et al., 2016). Dessa forma, o objetivo deste trabalho foi verificar, dentre os casos notificados na Rede Regional de Atenção à Saúde (RRAS) 11 (DRS Presidente Prudente), a ocorrência de Tuberculose na População Privada de Liberdade em comparação ao restante da população.

\section{METODOLOGIA}

Trata-se de um estudo transversal, quantitativo, utilizando registros do Sistema de Informação de Agravos de Notificação (SINAN) dos casos confirmados de Tuberculose, no Sistema de Informações de Saúde do SUS (DATASUS). Os dados coletados usaram como parâmetro a Macrorregião de Saúde de Notificação RRAS 11, no período entre janeiro de 2012 a maio de 2016. Por se tratar de dados secundários de domínio público, o estudo não necessitou de apreciação do Comitê de Ética em Pesquisa.

\section{RESULTADOS}

De acordo com o SINAN, na RRAS 11, no período de janeiro de 2012 a maio de 2016, foram notificados 1.832 casos confirmados. Dentre eles, 1.093 casos foram de pessoas privadas de liberdade, 736 não privadas de liberdade e 03 casos com essa situação ignorada, como observado no gráfico 1. 


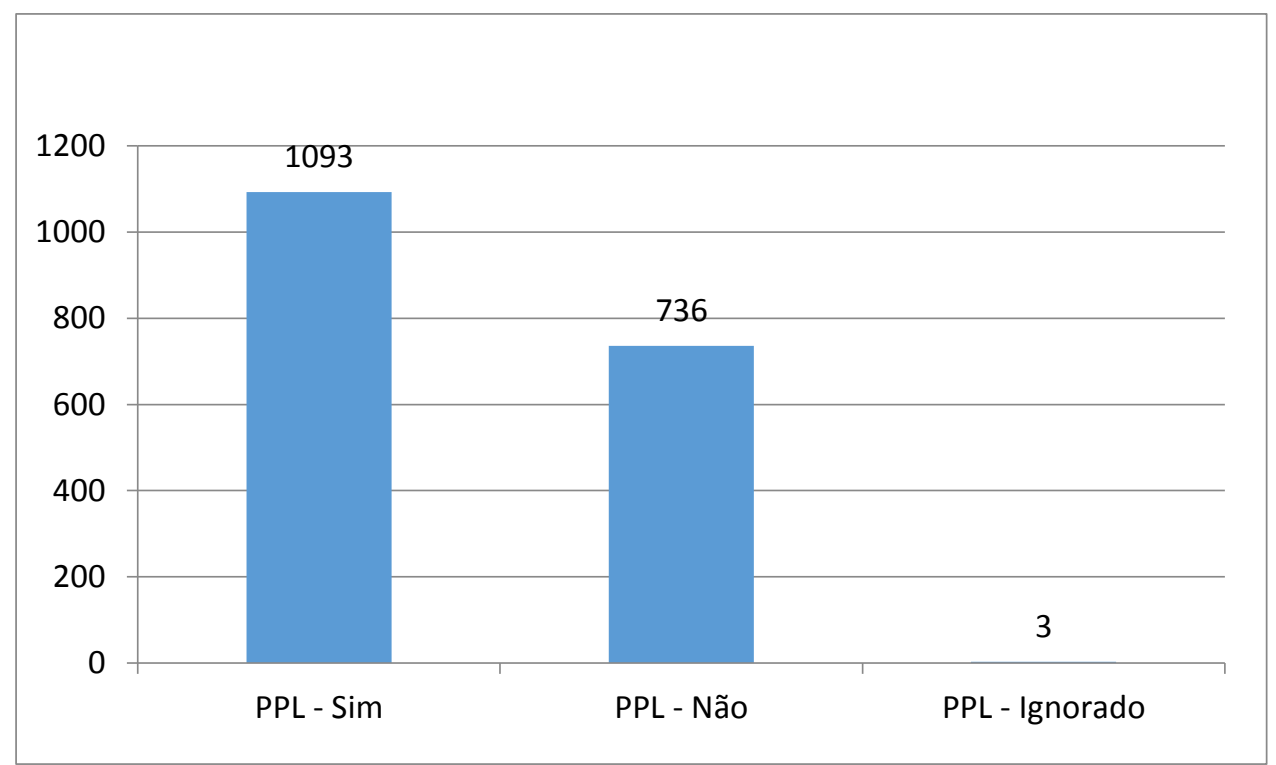

Gráfico 1. Tuberculose - casos confirmados notificados no Sistema de Informação de Agravos de Notificação - São Paulo de 2012 a 2016.

PPL: Ign/Branco, Sim, Não

Fonte: Ministério da Saúde/SVS - Sistema de Informação de Agravos de Notificação - Sinan Net

\section{Notas:}

1. Excluídos casos não residentes no Brasil ou encerrados com mudança de diagnóstico.

2. Períodos disponíveis ou período correspondem ao ano de notificação dos casos.

Dados atualizados em 25/05/2016.

Na população privada de liberdade, 1.081 dos casos foram masculinos e apenas 12 , femininos. Na população não privada de liberdade, 524 casos foram masculinos e 212 femininos. O que totaliza 1.608 casos masculinos (juntamente com os 03 casos com situação ignorada) e 224 casos femininos, demostrado no gráfico 2.

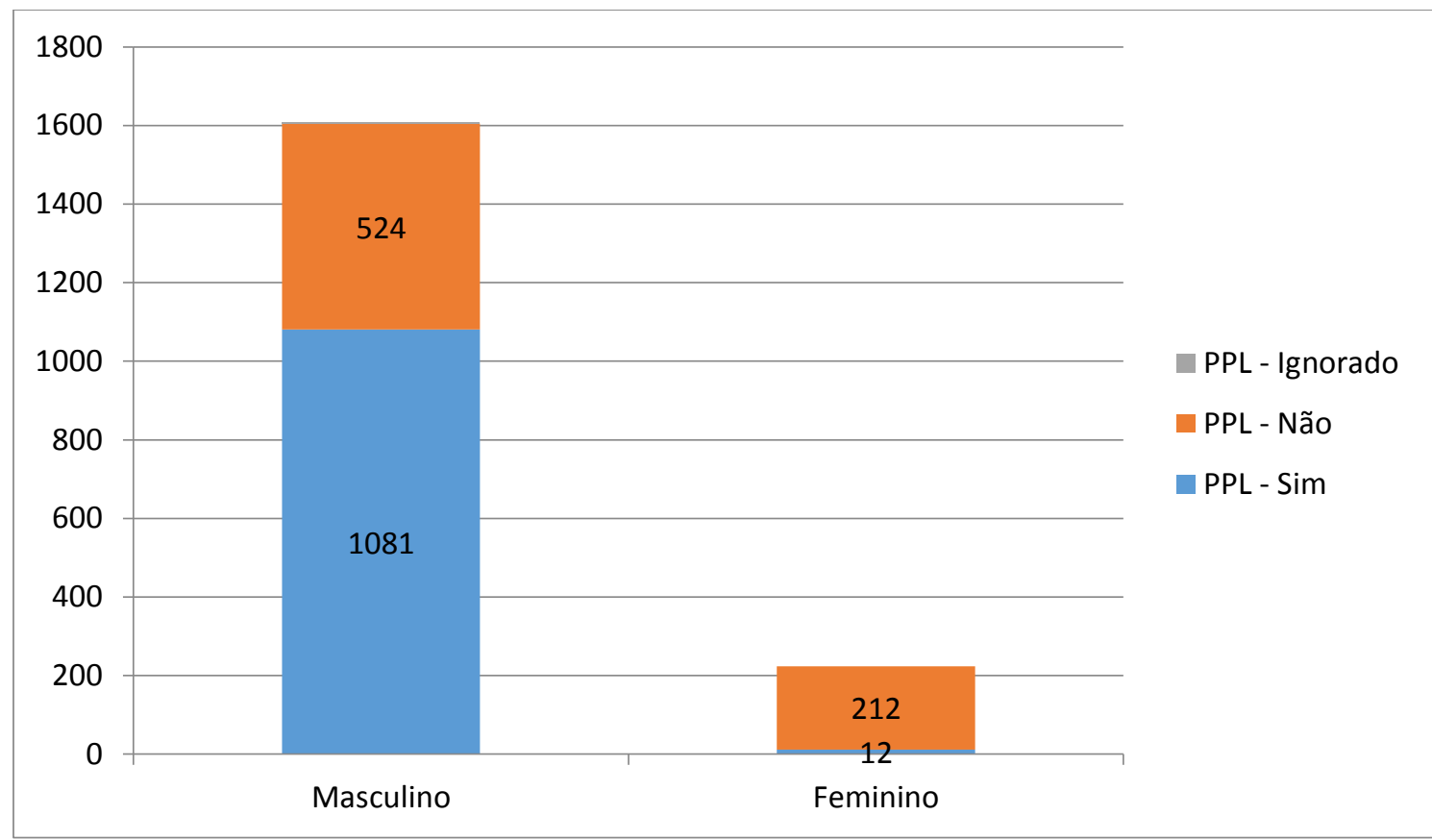

Gráfico 2. Tuberculose: casos confirmados por sexo segundo PPL no Sistema de Agravos de Notificação - São Paulo no período de 2012 a 2016.

PPL: Ign/Branco, Sim, Não

Fonte: Ministério da Saúde/SVS - Sistema de Informação de Agravos de Notificação - Sinan Net 
Notas:

1. Excluídos casos não residentes no Brasil ou encerrados com mudança de diagnóstico.

2. Períodos disponíveis ou período correspondem ao ano de notificação dos casos.

3. Dados atualizados em 25/05/2016.

Num total de 1.832 de casos relatados, 05 excluídos como ignorado /Branco, a faixa entre 25 a 34 anos concentra o maior número de casos no total, sendo que 633 deles são de pessoas privadas de liberdade, observável na tabela 1.

Tabela 1: Tuberculose - casos confirmados por Faixa Etária 7 segundo PPL notificados no Sistema de Informação de Agravos de Notificação - São Paulo no período de 2012 a 2016.

\begin{tabular}{c|c|c|c|c|c|c|c|c}
\hline $\begin{array}{c}\text { PPL: } \\
\begin{array}{c}\text { Ign/Branco, } \\
\text { Sim, } \\
\text { NãoPPL }\end{array}\end{array}$ & $\begin{array}{c}\text { Em } \\
\text { branco/IGN }\end{array}$ & $\begin{array}{c}0 \text { a } 14 \\
\text { anos }\end{array}$ & $\begin{array}{c}15 \text { a } \\
24 \\
\text { anos }\end{array}$ & $\begin{array}{c}25 \text { a } \\
34 \\
\text { anos }\end{array}$ & $\begin{array}{c}45 \text { a } \\
54 \\
\text { anos }\end{array}$ & $\begin{array}{c}55 \text { a } \\
64 \\
\text { anos }\end{array}$ & $\begin{array}{c}65 \text { e } \\
+\end{array}$ & Total \\
\hline Total & 5 & 18 & 314 & 785 & 476 & 122 & 112 & 1.832 \\
\hline Ign/branco & - & - & 1 & - & 2 & - & - & 3 \\
\hline Sim & 4 & 2 & 228 & 633 & 215 & 7 & 4 & 1.093 \\
\hline Não & 1 & 16 & 85 & 152 & 259 & 115 & 108 & 736 \\
\hline
\end{tabular}

Fonte: Ministério da Saúde/SVS - Sistema de Informação de Agravos de Notificação - Sinan Net

Notas:

1. Excluídos casos não residentes no Brasil ou encerrados com mudança de diagnóstico.

2. Períodos disponíveis ou período correspondem ao ano de notificação dos casos.

3. Dados atualizados em 25/05/2016.

\section{DISCUSSÃO}

Com base nos dados coletados, na Rede Regional de Atenção à Saúde 11, entre janeiro de 2012 e maio de 2016, foram identificados 1832 casos de TB, sendo 1093 diagnosticados na População Privada de Liberdade (PPL), representando 59,66\% dos casos. Dentre o total casos notificados, $87,77 \%$ eram do sexo masculino e a faixa etária que concentrou o maior número de casos foi entre 25 a 34 anos, representando $42,84 \%$.

Em relação apenas aos casos notificados em pessoas privadas de liberdade, percebeu-se que esta população é, em sua grande maioria, masculina - representando $98,90 \%$ dos casos - e na faixa etária entre 25 a 34 anos de idade $-57,91 \%$.

Dessa forma, a partir da análise dos dados é possível constatar que pessoas em situação prisional realmente constituem uma população de risco para Tuberculose na Macrorregião estudada. Embora representem uma minoria da população - no Brasil, a população privada de liberdade apresentou, em 2013, coeficiente de incidência de 985,3/100 mil hab -, agregam a maioria dos casos de Tuberculose (BOLETIM EPIDEMIOLÓGICO, 2015).

Ainda que esteja claro que há um problema de saúde pública em relação a TB e o sistema penitenciário, sua magnitude ainda é pouco conhecida. A introdução, em 2007, da informação sobre a origem prisional na ficha de notificação de TB permitiu uma primeira avaliação nacional e, em 2008, constatou-se que a população prisional, que representa apenas $0,2 \%$ da população do País, contribuiu com 5\% dos casos notificados em 2008 no Sistema de Informação de Agravos de Notificação - Sinan. (MANUAL DE RECOMENDAÇÕES PARA O CONTROLE DA TUBERCULOSE NO BRASIL, 2011). Em 2014, com o objetivo de fortalecer as ações de controle da tuberculose no sistema prisional, foi iniciada a implementação de atividades para o rastreamento de casos de tuberculose em alguns presídios no Brasil (BOLETIM EPIDEMIOLÓGICO, 2015). 
Alguns dos fatores que contribuem para a alta endemicidade da doença e sua transmissão nessa população são celas superpopulosas, mal ventiladas e com pouca iluminação solar, exposição frequente ao Mycobacterium tuberculosis em ambiente confinado, falta de informação sobre o problema e dificuldade de acesso aos serviços de saúde na prisão (BRASIL, 2011).

Além dessas condições de confinamento, a maior susceptibilidade da população carcerária inclui fatores de risco como: pobreza, abuso de substâncias, estilos de vida não saudáveis anteriores, nutrição inadequada e infecção por HIV (STUCKLER et al., 2008).

É importante ressaltar que a tuberculose na população privada de liberdade não é uma situação isolada, também traz riscos para as comunidades externas. Os presos podem infectar profissionais da saúde, guardas da prisão e seus cônjuges e filhos, e, muitas vezes, não se faz um acompanhamento satisfatório dos prisioneiros infectados após a sua libertação (STUCKLER et al., 2008).

\section{CONCLUSÃO}

A tuberculose continua sendo um desafio a ser superado pelo Brasil, principalmente no ambiente penitenciário. Porém, não é um problema isolado aos muros das penitenciárias, representa um problema de saúde pública geral, já que os surtos da prisão foram associados diretamente ao aumento da incidência de tuberculose na comunidade. Apesar da redução no coeficiente de incidência, a doença ainda é endêmica no país e na macrorregião RRAS11, se concentrando, sobretudo, em populações mais vulneráveis, como a população privada de liberdade do sexo masculino com idades entre 25 e 34 anos, necessitando de maiores cuidados nesse setor da sociedade.

\section{REFERÊNCIAS}

Lonnroth K, Castro KG, Chakaya JM, Chauhan LS, Floyd K, Glaziou P, et al. Tuberculosis control and elimination 2010-50: cure, care and social development. Lancet. 2010;375(9728):1814-29.

World Health Organization (WHO). Global tuberculosis control report. Genebra: WHO; 2011. (WHO/HTM/TB/2011.16), https://doi.org/10.1016/S0140-6736(10)60483-7.

BRASIL. Fundação Nacional de Saúde. Plano Nacional de Controle da Tuberculose. Ministério da Saúde, Brasília, 1999.

World Health Organization (WHO). Global Tuberculosis Control. WHO Report, WHO/TB/98.237, 1998

BRASIL. Ministério da Saúde. Secretaria de Vigilância em Saúde e Departamento de Vigilância Epidemiológica. Manual de recomendações para o controle da tuberculose no Brasil. Brasília, 2011.

Navarro, PD, et al. Prevalence of latent Mycobacterium tuberculosis infection in prisoners. Jornal Brasileiro de Pneumologia 42.5 (2016): 348-355, https://doi.org/10.1590/S1806-37562016000000001.

Stuckler, D, et al. Mass incarceration can explain population increases in TB and multidrugresistant TB in European and central Asian countries. Proceedings of the National Academy of Sciences 105.36 (2008): 13280-13285, https://doi.org/10.1073/pnas.0801200105.

Boletim Epidemiológico. Secretaria de Vigilância em Saúde - Ministério da Saúde, v.46, n.9, 2015. 